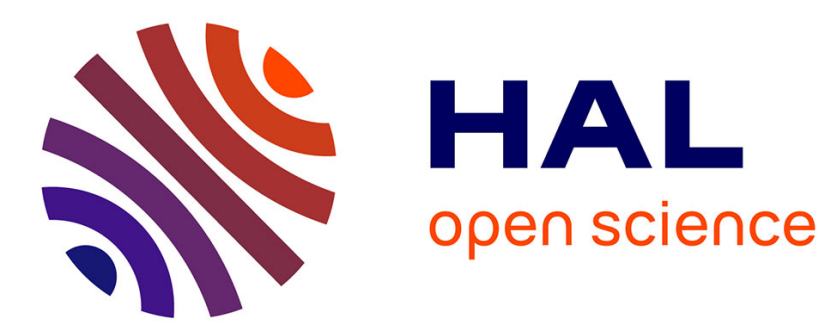

\title{
L'élevage en Guyane. Bilan des travaux réalisés sur les espèces domestiques et sur deux espèces sauvages autochtones
}

\author{
Stéphane Ingrand, G. Hostache
}

\section{To cite this version:}

Stéphane Ingrand, G. Hostache. L'élevage en Guyane. Bilan des travaux réalisés sur les espèces domestiques et sur deux espèces sauvages autochtones. Productions Animales, 1993, 6 (5), pp.319332. hal-00896065

\section{HAL Id: hal-00896065 https://hal.science/hal-00896065}

Submitted on 1 Jan 1993

HAL is a multi-disciplinary open access archive for the deposit and dissemination of scientific research documents, whether they are published or not. The documents may come from teaching and research institutions in France or abroad, or from public or private research centers.
L'archive ouverte pluridisciplinaire HAL, est destinée au dépôt et à la diffusion de documents scientifiques de niveau recherche, publiés ou non, émanant des établissements d'enseignement et de recherche français ou étrangers, des laboratoires publics ou privés. 
INRA Prod. Anim., 1993, 6 (5), 319 - 332.

\section{S. INGRAND, G. HOSTACHE*}

Départements Systèmes Agraires et Développement / Elevage

et Nutrition des Herbivores,

Laboratoire Adaptation des Herbivores aux Milieux, Theix, 63122 Saint-Genès

Champanelle

* Département Hydrobiologie-Faune sauvage, Campus de Beaulieu, 35042 Rennes Cedex
L'élevage en Guyane. Bilan des travaux réalisés sur les espèces domestiques et sur deux espèces sauvages autochtones

En 1965, l'installation en Guyane de la base spatiale de Kourou a engendré la coexistence, sur un petit territoire de $90000 \mathrm{~km}^{2}$, de cultures radicalement différentes : des indiens vivant de chasse et de pêche jusqu'aux technologies ultra-sophistiquées du spatial. Parallèlement aux efforts déployés autour de la fusée Ariane, des tentatives d'aide au développement d'autres secteurs d'activité économique se sont succédé, notamment en agriculture. La dernière en date, baptisé "Plan Vert" (1975), devait permettre d'atteindre l'autosuffisance alimentaire en matière de viande bovine. Depuis plus de 15 ans, cette opération a permis d'accumuler de nombreux résultats techniques, mais les objectifs assignés au plan n'ont jamais été réalisés.

\section{Résumé}

Les travaux à caractère zootechnique réalisés par l'TNRA sur sa station expérimentale dans le cadre du Plan Vert, ont surtout concerné l'élevage bovin à viande (zébu Brahman). Ils ont permis l'acquisition de résultats portant sur les performances de reproduction en milieu équatorial, et la conduite des taurillons à l'herbe entre le sevrage (âge de 9 à 10 mois) et l'abattage (âge de 24 à 30 mois). Parallèlement, entre 1985 et 1987, des suivis de troupeaux privés ont permis l'analyse des résultats techniques des élevages locaux, ainsi que l'ébauche d'une typologie des exploitations basée sur les pratiques des éleveurs.

Plusieurs enquêtes, ainsi que des études par filières (porcine, avicole, cunicole) ont également montré que, relativement à l'ensemble de l'activité agricole, la production de viande bovine était loin d'être prédominante en Guyane. Une caractéristique importante des exploitations pratiquant l'élevage s'est avérée être la diversification de leurs spéculations, gage de souplesse, de capacité d'adaptation au marché et aux besoins de trésorerie, et donc de leur pérennité.

D'autre part, l'élevage de certaines espèces sauvages autochtones pourraient, dans ce contexte de diversification des spéculations, constituer une alternative intéressante par la valorisation des ressources biologiques naturelles de la Guyane. Deux espèces ont déjà fait l'objet d'études : un mammifère rongeur, le cabiaï (Hydrochoerus hydrochaeris) et un poisson d'eau douce, l'atipa (Hoplosternum littorale).

Illustrées par ces exemples, quelques pistes de réflexion sont présentées concernant les conditions d'émergence de systèmes d'élevages pérennes en Guyane. Elles soulignent notamment l'importance de la prise en compte des aspects autres que techniques (principalement économiques et sociologiques) pour l'appui au développement de l'élevage dans le contexte très particulier de la Guyane. 
La Guyane francaise, territoire de 90000 $\mathrm{km} 2$ situé entre le Brésil et le Surinam (voir carte), a le statut de département français depuis 1946. Sa population était estimée à 120000 habitants en 1990 . C'est en 1975 qu'a été initiée par l'Etat français "l'opération Guyane" qui visait à redynamiser l'économie du territoire sur la base d'une immigration importante et qui devait aboutir à la création de 5000 emplois dans les secteurs de l'agriculture et de la forêt et 5000 autres emplois dans les secteurs de l'industrie du bois, des mines et des services. A cette époque, la population totale de la Guyane était de 55000 habitants et 3750 hectares étaient cultivés, dont plus de $60 \%$ sous forme de cultures itinérantes sur brûlis (abattis). Le cheptel bovin comptait environ 2000 têtes, assurant $5 \%$ de la consommation locale. "L'opération Guyane", baptisée ultérieurement "Plan Vert" en raison de la limitation des programmes aux aspects agricoles, devait initialement permettre à échéance de 5 ans la constitution d'un troupeau bovin assurant une production annuelle de viande de 500 tonnes ( $50 \%$ des besoins en 1975), et de 1000 tonnes au bout de 10 ans avec 30000 têtes de bétail. Finalement, en 1987, la production n'a atteint que 350 tonnes et couvrait alors $25 \%$ des besoins locaux.

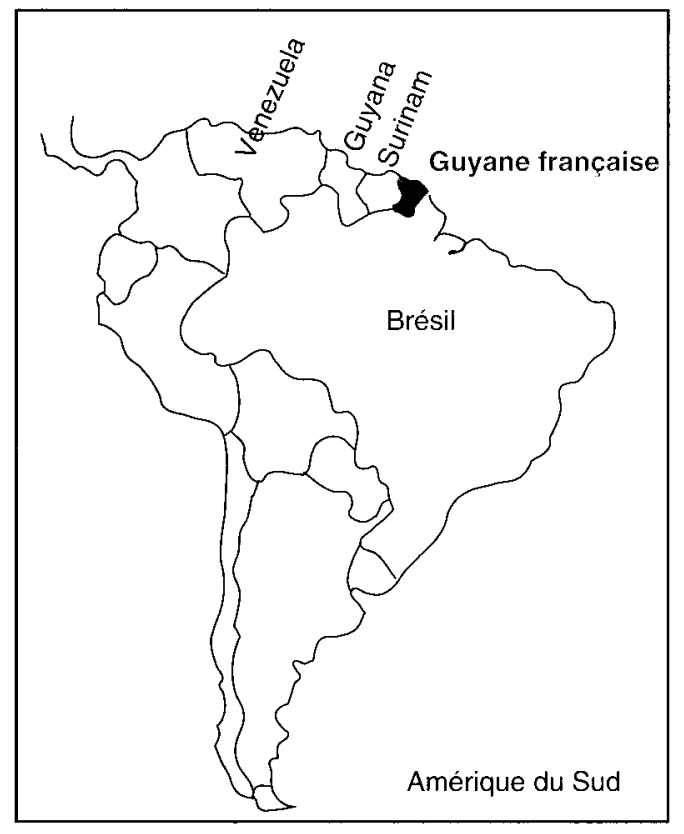

Pour plus d'informations sur la mise en place du Plan Vert et sur les modalités d'intervention des structures de Recherche françaises dans ce contexte, on pourra se référer aux nombreux textes et ouvrages s'y rapportant (INRA 1981, INRA 1985 Gachet 1989, Vissac 1992).

L'objet de cet article est de présenter dans un premier temps les résultats des recherches à caractère zootechnique entreprises par le Département Systèmes Agraires et Développement de l'INRA en Guyane, sur les espèces bovines dans le cadre du Plan-Vert et sur d'autres espèces domestiques : avicoles, porcines et cunicoles. Dans un second temps sera dressé l'état des premières études entreprises sur des espèces autochtones susceptibles d'être valorisées par l'élevage (cabiaï et atipa), ceci dans le but d'évaluer les possibilités d'exploitation des ressources biologiques naturelles de la Guyane. Enfin, nous tenterons d'exposer les points importants à prendre en compte pour conforter les acquis et surtout pour contribuer à l'élaboration de systèmes d'élevages "durables" dans le contexte particulier de la Guyane : points liés à la biologie des espèces, à l'économie du territoire ou encore à son contexte sociologique.

\section{1 / La production bovine}

Les résultats acquis en matière d'élevage bovin allaitant en Guyane sont issus de trois types de démarche : suivis d'élevages privés, expérimentations conduites sur le domaine de l'INRA concernant l'engraissement des taurillons à l'herbe et analyse des résultats techniques de fermes d'Etat, permise par les nombreux enregistrements effectués sur une longue période.

\section{1 / Les suivis d'élevages privés}

Les suivis d'élevages, d'abord limités à 7 exploitations, ont ensuite été étendus à un plus grand nombre de cas (21), l'objectif étant qu'ils soient pris en charge progressivement par les agents du Développement. La vocation de ces suivis était de fournir des références techniques, d'identifier les différents systèmes d'élevage bovin existant en Guyane (typologie) et d'influer sur la définition des thèmes de travail pour la Recherche. Quelques caractéristiques des 21 exploitations suivies en 1986 sont présentées au tableau 1. L'ensemble pratiques/performances a été analysé pour l'année 1986 (Lebouteiller et al 1987) et 4 groupes d'éleveurs avaient alors été distingués :

Groupe $1(n=7)$ : éleveurs ayant adopté un modèle de production intensif (fertilisation, chargement, allotement), associé à d'excellents résultats techniques.

Groupe $2(n=2)$ : modèle de production plus extensif (chargement plus faible, moindre fertilisation, taille des lots d'animaux plus élevée), avec également de bons résultats techniques grâce à une bonne connaissance du milieu, des animaux et une main-d'oeuvre compétente.

Groupe $3(n=5)$ : chargement le plus élevé de l'échantillon (supérieur à 5 UBT*/ha), associé à une faible fertilisation et à un mauvais état des prairies. Ces éleveurs étaient en difficulté, avec des problèmes dus au chargement trop important.

Groupe $4(n=7)$ : exploitations ayant du mal à atteindre le régime de croisière (entrée en production très tardive par rapport à l'arrivée des premiers animaux). Ces éleveurs étaient en phase d'apprentissage et d'adaptation de leurs exploitations aux contraintes du milieu. 
Tableau 1. Quelques caractéristiques des 21 élevages bovins-viande du réseau guyanais (1986).

\begin{tabular}{|c|c|c|c|c|c|c|c|c|c|c|}
\hline \multirow[t]{2}{*}{ Installation } & \multirow{2}{*}{$\begin{array}{c}\text { Arrivée } \\
\text { des premiers } \\
\text { animaux }\end{array}$} & \multirow{2}{*}{$\begin{array}{c}\text { Effectif } \\
\text { du cheptel } \\
\text { bovin }\end{array}$} & \multirow{2}{*}{$\begin{array}{c}\text { Effectif } \\
\text { vaches } \\
\text { reproductrices }\end{array}$} & \multirow{2}{*}{$\begin{array}{c}\text { Origine } \\
\text { des sols } \\
(1)\end{array}$} & \multirow{2}{*}{$\begin{array}{c}\text { Surface en } \\
\text { prairies } \\
\text { (ha) }\end{array}$} & \multirow{2}{*}{$\begin{array}{c}\text { \% moyen } \\
\text { recouvr. } \\
(2)\end{array}$} & \multicolumn{3}{|c|}{$\begin{array}{c}\text { Fertilisation } \\
(\mathrm{kg} / \mathrm{h} / \mathrm{an})\end{array}$} & \multirow{2}{*}{$\begin{array}{c}\text { N/ha de } \\
\text { surface } \\
\text { totale }\end{array}$} \\
\hline & & & & & & & $\mathrm{N}$ & $\mathrm{P}$ & $\mathrm{K}$ & \\
\hline \multirow[t]{4}{*}{1976} & 1977 & 502 & 187 & forêt & 147 & 79 & 19 & 77 & 72 & 19 \\
\hline & 1977 & 820 & 299 & forêt & 292 & 44 & 60 & 56 & 56 & 60 \\
\hline & 1977 & 270 & 156 & forêt & 60 & $<20$ & & & & \\
\hline & 1978 & 143 & 0 & forêt/savane & 37 & 72 & 108 & 57 & 66 & 108 \\
\hline \multirow[t]{5}{*}{1977} & 1977 & 592 & 298 & forêt & 200 & 57 & & & & \\
\hline & 1978 & 537 & 299 & forêt & 135 & 82 & 10 & 31 & 38 & 9 \\
\hline & 1979 & 162 & 88 & forêt/savane & 40 & 38 & 17 & 28 & 28 & 3 \\
\hline & 1979 & 496 & 285 & forêt/savane & 157 & 80 & 80 & 70 & 70 & 80 \\
\hline & 1979 & 520 & 302 & forêt & 147 & 51 & 8 & 41 & 52 & 8 \\
\hline \multirow[t]{4}{*}{1979} & 1980 & 142 & 75 & forêt & 37 & 33 & 90 & 31 & 31 & 37 \\
\hline & 1981 & 33 & 19 & forêt & 20 & 52 & 0 & 0 & 0 & 0 \\
\hline & 1981 & 364 & 203 & forêt & 145 & $<20$ & 42 & 42 & 42 & 29 \\
\hline & 1982 & 156 & 85 & savane & 126 & 27 & 72 & 39 & 40 & 58 \\
\hline 1981 & 1984 & 11 & 9 & forêt & 6 & 26 & & & & \\
\hline \multirow[t]{4}{*}{1982} & 1982 & 81 & 56 & savane & 70 & 29 & & & & \\
\hline & 1983 & 382 & 134 & savane/forêt & 145 & 82 & 59 & 65 & 65 & 59 \\
\hline & 1984 & 260 & 55 & forêt & 64 & 78 & 76 & 48 & 65 & 76 \\
\hline & 1985 & 25 & 0 & savane/forêt & 18 & 66 & 70 & 73 & 74 & 70 \\
\hline 1983 & 1985 & 48 & 22 & forêt & 18 & 75 & 93 & 93 & 93 & 40 \\
\hline \multirow[t]{2}{*}{1984} & 1984 & 29 & 0 & forêt & 13 & 82 & 104 & 74 & 74 & 104 \\
\hline & 1985 & 87 & 48 & forêt & 29 & 39 & 50 & 50 & 50 & 50 \\
\hline
\end{tabular}

(1) type de sol et de couvert avant l'implantation des prairies

(2) pourcentage de recouvrement des parcelles en espèces cultivées

En 1988 seuls trois ou quatre élevages étaient visités par les deux conseillers agricoles chargés de ce travail et aucun résultat exploitable n'a pu en être retiré, tant en 1987 qu'en 1988. L'intérêt des éleveurs se focalisant alors uniquement sur les aspects financiers liés à leur niveau d'endettement, il n'est pas apparu opportun de continuer cette opération.

Le suivi s'est maintenu pour deux fermes expérimentales d'Etat d'où sont issues les références acquises ces cinq dernières années en matière de conduite des taurillons à l'herbe entre le sevrage et l'abattage (Ingrand et al 1993a), de performances de reproduction des femelles (Ingrand et al 1993b) et de conduite d'élevage (Ingrand et al 1993c). Concernant les suivis d'élevages privés, la désagrégation du réseau mis en place ne semble pas seulement due à la crise de l'élevage bovin, mais également aux carences méthodologiques et structurelles de l'encadrement direct des éleveurs avec en outre un déphasage permanent entre l'apparition des problèmes sur le terrain et l'obtention des références et résultats supposés aptes à les résoudre.

\section{2 / Engraissement des taurillons à l'herbe}

Les essais à caractère purement zootechnique conduits sur le domaine de l'INRA se sont déroulés de 1985 à 1990.
Chronologiquement, ils ont concerné :

- le niveau et le rythme de complémentation pour une production rapide et en quantité suffisante après la mise en valeur des terres et les investissements en animaux et en matériel ;

- les rythmes d'exploitation, la nature de l'herbe utilisée et le chargement afin de gérer le pâturage de façon optimale ;

- les effets de la castration et du croisement en vue d'obtenir une viande de qualité comparable à celle de la viande importée.

Les caractéristiques et les principaux résultats de ces essais sont rassemblés dans le tableau 2 et ont été publiés par ailleurs (Ingrand et al 1993a).

Depuis 1988, les études ont été orientées vers la ressource fourragère et la simplification de sa gestion. Trois essais destinés à tester une méthode d'exploitation des pâturages comparable au pâturage intensif libre des milieux tempérés ont ainsi été mis en place, avec des résultats techniques comparables à ceux obtenus avec les sytèmes en rotation (Béreau et al 1993).

\section{3 / Bilan des études sur la reproduction}

Outre les résultats issus des suivis d'élevages privés, nous avons pu disposer des don- 
Tableau 2. Caractéristiques et principaux résultats concernant les essais d'engraissement de taurillons zébus conduits à l'herbe au domaine INRA de Combi (Guyane française). Essai 1 : du 19/11/84 au 15/01/86, essai 2 : du 22/07/85 au 29/08/86, essai 3 : du 21/08/85 au 21/12/86, essai 4 : du 18/06/86 au 21/07/87, essai 5 : du 24/02/87 au 27/07/88, essai 6 : du 19/11/87 au 22/09/89.

\begin{tabular}{|c|c|c|c|c|c|c|c|c|c|c|c|c|c|c|}
\hline \multirow{2}{*}{ Essai } & \multicolumn{6}{|c|}{ Animaux } & \multicolumn{5}{|c|}{ Alimentation } & \multicolumn{3}{|c|}{ Production } \\
\hline & $\mathrm{Nb}$ & $\begin{array}{c}\text { Age début } \\
\text { (j) }\end{array}$ & $\begin{array}{c}\text { Pás début } \\
(\mathrm{kg})\end{array}$ & $\begin{array}{c}\text { Age fin } \\
\text { (j) }\end{array}$ & $\begin{array}{c}\text { Poids fin } \\
\text { (kg) }\end{array}$ & $\begin{array}{l}\text { Entier } \\
\text { Castré }\end{array}$ & \multicolumn{2}{|c|}{ Complémentattion } & $\begin{array}{l}\text { Nature } \\
\text { herbe }^{* *}\end{array}$ & $\begin{array}{l}\text { Age } \\
\text { rep (j) }\end{array}$ & $\begin{array}{c}\text { Chargt } \\
\text { (têtesha) }\end{array}$ & $\begin{array}{c}\text { Gain total } \\
(\mathrm{kg})\end{array}$ & $\begin{array}{c}\text { GMQ } \\
\text { (g) }\end{array}$ & $\begin{array}{l}\text { Gain } \\
\text { (kgha) }\end{array}$ \\
\hline 1. Niveau & 16 & $356: 72$ & $19611^{2}$ & $808 \pm 72$ & $412 \pm 25$ & E & $01,1)$ & Constant & mixte & 35 & 4,5 & $217 \pm 21$ & $514 \pm 58$ & 844 \\
\hline & 18 & $40 ; 0,69$ & $19 x \div 1: 3$ & $794 \pm 89$ & $440 \pm 22$ & $\mathrm{E}$ & 1,4 & Constant & mixte & 35 & 5,5 & $242 \pm 21$ & $619 \pm 55$ & 1243 \\
\hline complé. & 19 & 517111 & $26 \div-39$ & $874 \pm 111$ & $438 \pm 38$ & $\mathrm{E}$ & 1)., 3 & Constant & mixte & 35 & 3,4 & $176 \pm 51$ & $490 \pm 81$ & 608 \\
\hline mentation & 20 & $51 i=114$ & $261-29$ & $832 \pm 114$ & $446 \pm 38$ & $\mathrm{E}$ & 1.9 & Constant & mixte & 35 & 3,8 & $186 \pm 44$ & $607 \pm 84$ & 842 \\
\hline 2-Vitesse de & 21 & $383+74$ & $216 \pm 35$ & $786 \pm 74$ & $458 \pm 36$ & E & 1,0 & Constant & swaz & 1 & 4,0 & $241 \pm 38$ & $599 \pm 68$ & 908 \\
\hline rotation & 21 & $398 \pm 87$ & $213 \pm 42$ & $791+87$ & $423 \pm 53$ & $\mathrm{E}$ & 1,0 & Constant & swaz & $31]$ & 4,0 & $210 \pm 47$ & $534 \pm 69$ & 818 \\
\hline 3-Rythme de & & & & & & & & & & & & & & \\
\hline complé- & 22 & $298 \pm 56$ & $189 \pm 22$ & $781 \pm 56$ & $396 \pm 38$ & E & $20, \%$ & Bariable & mixte & 25 & 5,5 & $207 \pm 28$ & $429+62$ & 861 \\
\hline mentation & 22 & $295 \pm 55$ & $188+25$ & $778 \pm 55$ & $419 \pm 40$ & $\mathrm{E}$ & $11)$ & Content & mixte & 25 & 5,8 & $231 \pm 30$ & $478 \pm 63$ & 1012 \\
\hline 4- Nature du & 19 & $367 \pm 114$ & $240 \pm 51$ & $763 \pm 114$ & $457 \pm 61$ & $\mathrm{E}$ & 1,0 & Constant & swal & 31 & 4,7 & $217 \pm 29$ & $543 \pm 65$ & 931 \\
\hline pâturage & 20 & $378 \pm 89$ & $239+49$ & $774+89$ & $404 \pm 54$ & E & 1,0 & Constant & der. & 41 & 4,0 & $165 \pm 28$ & $412 \pm 59$ & 601 \\
\hline 5-Vitesse de & 19 & $373+40$ & $237 \pm 25$ & $940 \pm 40$ & $441 \pm 36$ & E & 0,3 & Constant & mixte & 28 & 4.5 & $204 \pm 28$ & $394 \pm 55$ & 647 \\
\hline rotation et & 18 & $380 \pm 41$ & $235 \pm 23$ & $947 \pm 41$ & $436 \pm 29$ & $\mathrm{E}$ & 0,3 & Constant & mixte & 12 & 4.5 & $203 \pm 27$ & $392 \pm 51$ & 644 \\
\hline chargement & 22 & $383 \pm 45$ & $236+26$ & $950 \pm 45$ & $436 \pm 4.3$ & E & 0,3 & Constant & mixte & 28 & 60 & $200 \pm 28$ & $387 \pm 54$ & 847 \\
\hline 6. Castration & 7 & :HU14: & $1 x_{i}{ }_{i}$ & $1012 \pm 45$ & $428 \pm 51$ & E: & 0,3 & Constant & mixte & 32 & & $241 \pm 38$ & $359 \pm 57$ & 590 \\
\hline & 7 & 46928 & $223-12$ & $1141 \pm 27$ & $443 \pm 59$ & E: & 0,3 & Constant & mixte & 32 & & $249 \pm 31$ & $370 \pm 47$ & 608 \\
\hline & 5 & $(y)=43$ & $175^{-18}$ & $1005 \pm 43$ & $409+34$ & $f^{\prime}$ & 0,3 & Constant & mixte & 32 & 4,5 & $234 \pm 27$ & $348 \pm 40$ & 572 \\
\hline & 9 & $15 \times 32$ & $290-24$ & $1130+32$ & $470+28$ & c & 0,3 & Constant & mixte & 32 & & $244 \pm 28$ & $363 \pm 42$ & 596 \\
\hline
\end{tabular}

L'objectif de croissance de $500 \mathrm{~g} / \mathrm{j}$ est atteint seulement si la complémentation est importante ou si la pâturage est de bonne qualité et bien exploité.

* $2 \mathrm{~kg} /$ animal $/ \mathrm{j}$ puis $0,3 \mathrm{~kg} / \mathrm{animal} / \mathrm{j}$

** swaz = Digitaria swazilandensis $;$ déc. $=$ Brachiaria decumbens

**** temps de repousse $(\mathrm{j})$

nées enregistrées dans deux fermes d'Etat: une (Saint-Elie) pour la race zébu Brahman (photo 1) et l'autre (Matoury) pour la race Santa-Gertrudis (Ingrand et Jérôme 1993).
L'analyse des performances de reproduction de ces troupeaux est très importante car cet aspect conditionne actuellement très fortement les résultats économiques des élevages.

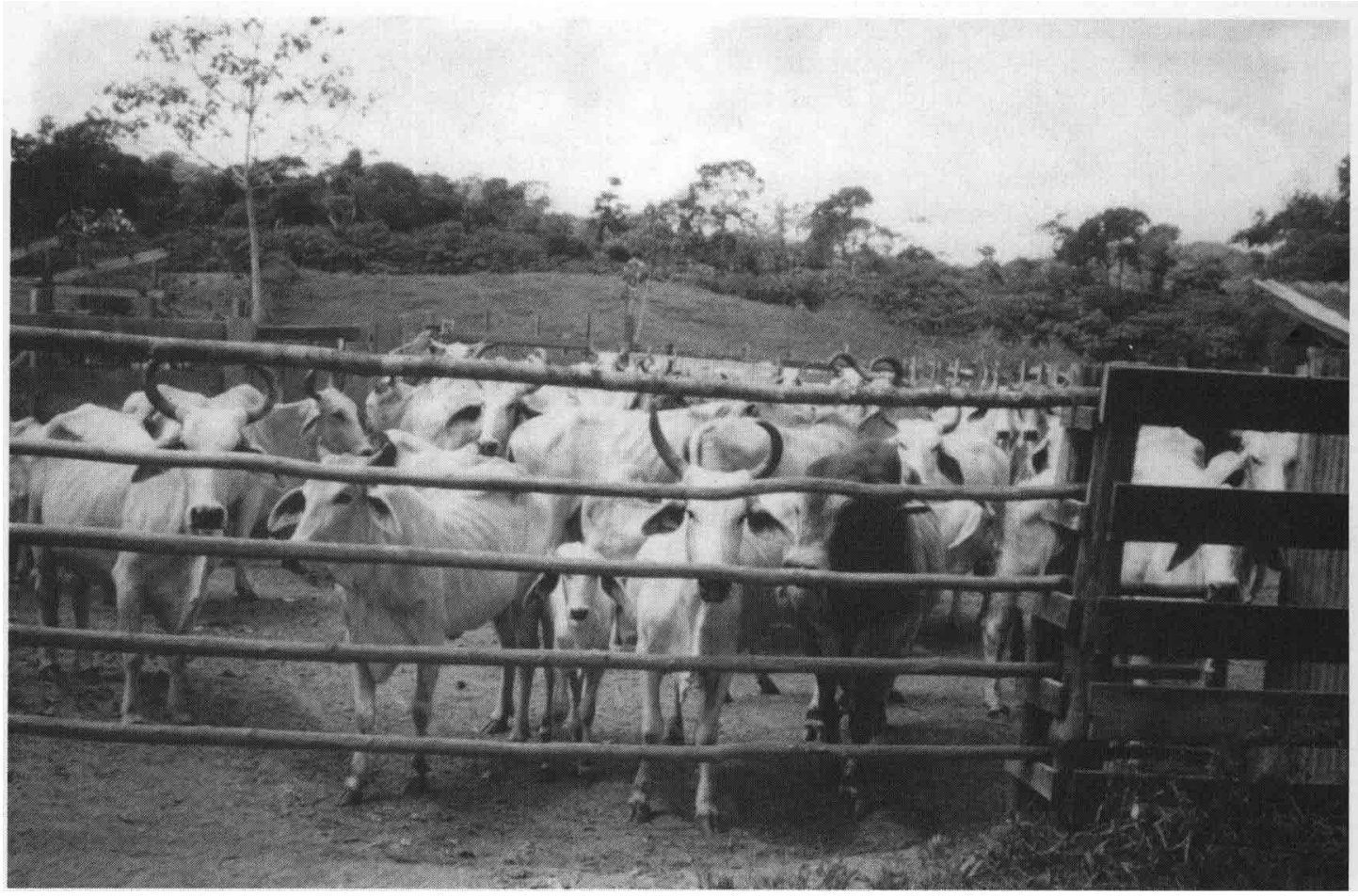

Troupeau zébu de race Brahman (cliché INRA/S. Ingrand) 
Dans les conditions favorables qui sont celles des fermes d'Etat en matière de suivi et de soins aux animaux, on n'observe pas d'influence significative des saisons sur la durée des cycles de reproduction et sur la répartition des vêlages sur l'année (exemple de SaintElie : figure 1). Les paramètres sensibles aux saisons sont plutôt la mortalité des produits (entre 0 et 3 mois d'âge) et les performances de croissance dans la phase de post-sevrage. Il semble qu'une conduite différentielle des lots d'animaux en fonction de leur état physiologique et selon les saisons soit plus adaptée à l'amélioration des paramètres du rendement des troupeaux qu'un calage des vêlages sur des périodes précises. La moyenne de 658 intervalles entre vêlages enregistrés à Saint-Elie est de 14 mois ( \pm 91 jours) ; cet intervalle varie fortement selon le rang de vêlage des animaux (figure 2). Un aspect important qui reste à approfondir concerne les conditions de mise à la reproduction des génisses, en termes d'âge et de poids.

Depuis 1986 , certains éleveurs ont pratiqué des croisements entre les zébus importés dans le cadre du Plan Vert et la race limousine, que ce soit par insémination ou par importation de reproducteurs. Cette pratique se conçoit pour des situations où la conduite d'élevage est bien maîtrisée et où une amélioration des qualités bouchères des produits destinés à la vente peut constituer un atout dans le cadre d'un marché de plus en plus demandeur (surtout face à la concurrence de la viande importée). Il apparaît nécessaire de quantifier précisément le gain de productivité ainsi procuré. En outre, il reste à savoir comment gérer la pratique de ce type de croisement en Guyane, tout particulièrement avec un troupeau zébu de base à effectif réduit. Un programme d'insémination a été initié dans ce cadre sur le troupeau de la ferme gérée par l'INRA, avec deux objectifs principaux :

- caractérisation de la croissance jusqu'au sevrage pour les deux sexes (veaux zébus : tableau 3), entre le sevrage et l'abattage pour les mâles, entre le sevrage et la mise à la reproduction pour les génisses ;

- constitution d'un troupeau synthétique croisé, composé d'une cinquantaine de mères en 1995.

Tableau 3. Croissance des veaux zébus sous la mère à Saint-Elie (Guyane).

\begin{tabular}{|lccccc|}
\hline Année & $\mathbf{1 9 8 4}$ & $\mathbf{1 9 8 5}$ & $\mathbf{1 9 8 6}$ & $\mathbf{1 9 8 7}$ & $\mathbf{1 9 8 8}$ \\
\hline Veaux mâles & & & & & \\
Poids à la naissance $(\mathrm{kg})$ & 30 & 29 & 29 & 28 & 28 \\
Poids au sevrage $(\mathrm{kg})$ & 166 & 176 & 173 & 163 & 190 \\
Age au sevrage $(\mathrm{j})$ & 239 & 237 & 247 & 250 & 258 \\
Croît naissance-sevrage $(\mathrm{g} / \mathrm{j})$ & 587 & 625 & 594 & 554 & 622 \\
\hline Veaux femelles & & & & & \\
Poids à la naissance $(\mathrm{kg})$ & 27 & 26 & 27 & 26 & 26 \\
Poids au sevrage $(\mathrm{kg})$ & 161 & 167 & 170 & 170 & 189 \\
Age au sevrage $(\mathrm{j})$ & 297 & 256 & 265 & 267 & 283 \\
Croît naissance-sevrage $(\mathrm{g} / \mathrm{j})$ & 502 & 564 & 546 & 536 & 588 \\
\hline
\end{tabular}

Figure 1. Répartition mensuelle des vêlages dans le troupeau de la ferme de Saint-Elie (Guyane). Moyenne sur 6 ans (1984-1989).

Vêlages mensuels (\%)

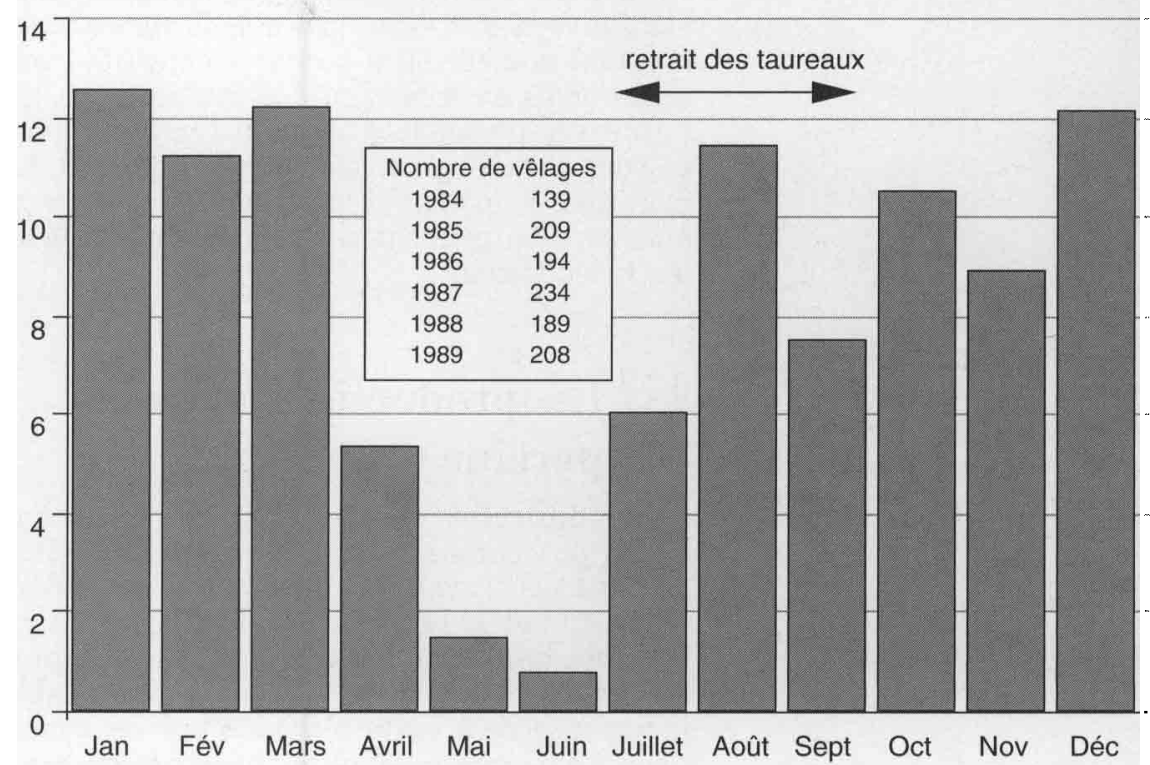

Figure 2. Evolution de l'intervalle entre deux vêlages en fonction du rang de vêlage dans le troupeau zébu Brahman de la ferme de Saint-Elie (Guyane)

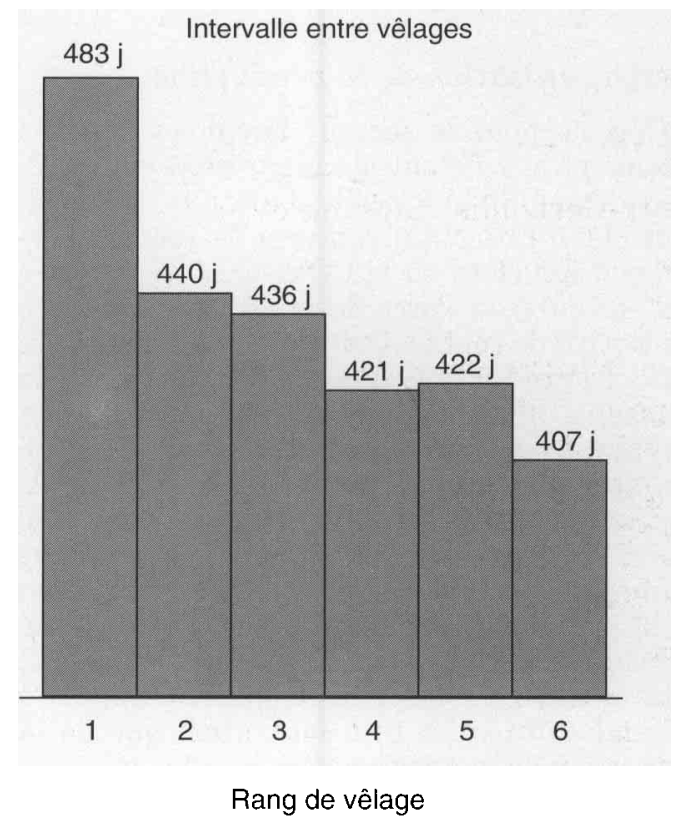

\section{4 / Perspectives}

Depuis 1986, les élevages issus du Plan Vert et qui ont su résister tant bien que mal aux périodes difficiles résultant de l'arrêt des aides financières, de la cessation d'activité de la coopérative et de toute forme d'organisation collective en général, ont dû s'adapter au cas par cas pour pérenniser leur activité (Gachet et al 1989). En 1991, les effectifs bovins étaient évalués à 14000 têtes pour l'ensemble de la Guyane et le souci actuel est de mettre 
en place des formules de gestion du cheptel au niveau collectif permettant de sauvegarder le niveau génétique des zébus Brahman, surtout après les opérations de décapitalisation qui ont eu lieu ces dernières années. Le contexte redevient favorable à l'émergence de formes d'organisation des éleveurs autour d'objectifs communs, mais en respectant les itinéraires et les initiatives personnels, dans la limite où elles ne vont pas à l'encontre de l'intérêt général. Le maintien du potentiel zébu en Guyane et son amélioration peuvent constituer alors un leitmotiv fédérateur.

\section{2 / Les productions avicole, porcine et cunicole}

Les difficultés rencontrées dans le secteur bovin ont conduit la station INRA-SAD à étendre ses travaux à l'ensemble des productions animales. La connaissance la plus large possible de toutes les formes d'élevage présentes en Guyane paraissait alors le préalable indispensable à toute réflexion sur les conditions d'émergence de systèmes agricoles autonomes et viables. Outre les enquêtes exhaustives réalisées dans différentes régions de la Guyane et concernant toutes les formes d'activité agricole, des actions à caractère plus spécifiquement zootechnique ont été entreprises.

\section{1 / Les filières avicole et porcine}

\section{a / Organisation de la production}

Comme pour le secteur bovin, les filières porcine et avicole ont été organisées en coopérative ou en SICA (Société d'Intérêts Collectifs Agricoles). En ce qui concerne le secteur porcin, il y a d'abord eu la COPORG (Coopérative des éleveurs de Porcs de la Guyane), créée à l'initiative de sept éleveurs. En 1980, la société PAPPI (Production Associée de Porcs et de Poissons en Intégration) s'est installée en Guyane avec pour vocation l'exportation de viande de porcs vers les Antilles et les USA. Les installations étaient prévues pour 540 truies, la fabrication d'aliments à partir de manioc cultivé sur place et la transformation de la viande (atelier de découpe-salaison). Les difficultés rencontrées pour conduire le projet à la mesure de ses ambitions ont abouti à l'abandon du volet piscicole, ainsi que de la production de manioc.

Les exportations s'avérant également problématiques (veto des USA pour des raisons sanitaires, concurrence des producteurs locaux aux Antilles), la production de la PAPPI est rapidement déversée sur le marché guyanais, concurrençant directement la COPORG. Les deux structures (PAPPI et COPORG) vont fusionner en 1985 avec la coopérative bovine pour former la SICAVIG, dont les résultats financiers restent négatifs jusqu'en novembre 1987, date de son dépôt de bilan. Suivent alors la CEBG (Coopérative des Eleveurs Bovins de Guyane) le 5 janvier 1988 et la COPORG en mai de la même année. Pour éviter la désorganisation totale du marché, une autre SICA est mise sur pied : la SICA Guyane-Viande, ayant pour objet l'abattage, la transformation et la mise en marché de viande bovine et porcine. Finalement, l'approvisionnement en aliment devenant aléatoire, la production s'en ressent et le marché n'est plus suffisamment pourvu. Les pores sont abattus de plus en plus jeunes $(36,6 \mathrm{~kg}$ de poids moyen des carcasses au cours du premier semestre 1989). La dissolution judiciaire est prononcée le 1er mars 1989.

Le secteur avicole a connu beaucoup moins de vicissitudes dans la mesure où la première forme réelle d'organisation n'est apparue qu'en 1987 avec la création de la Coopérative Avicole et Cunicole de Guyane (CACG). Là encore les projets étaient ambitieux, incluant la création d'un centre collectif de conditionnement des oeufs, d'un abattoir et la modernisation des élevages. Le manque de cohésion entre les adhérents de la coopérative n'a pas permis la concrétisation de ce programme. Actuellement, la CACG existe toujours et des budgets ont été accordés pour le recrutement d'un technicien qui est chargé, outre les aspects purement techniques, de réfléchir aux solutions de commercialisation en commun de la production des adhérents. Des subventions ont également été accordées pour l'amélioration technique des élevages ainsi que pour le suivi sanitaire.

\section{b / Situation des producteurs et organisation des marchés}

Les élevages avicoles et cunicoles se différencient de la même façon que les autres productions du secteur agricole en Guyane : il existe d'une part des élevages spécialisés, intégrés dans les circuits officiels d'aide et de financement et assurant la majeure partie de la production locale, d'autre part des élevages dits "traditionnels", intégrés dans des systèmes de production diversifiés associant spéculations animales et végétales, et le plus souvent en marge des systèmes d'aide (Bouilly 1989).

Les formes d'organisation décrites dans le paragraphe précédent et qui ont jalonné l'histoire des fillières avicole et porcine, concernent la première catégorie. Ces éleveurs, notamment en élevage porcin, ont été durement éprouvés par les différentes crises qui se sont succédé. Ils représentent une forme d'activité agricole fragile car dépendante de nombreux facteurs externes à l'exploitation et non maîtrisés (ou mal maîtrisés) : approvisionnement en animaux de renouvellement et en intrants divers. Seuls quelques uns ont su "court-circuiter" les fournisseurs locaux et traiter directement avec la métropole. C'est par ce biais qu'ont émergé les trois ou quatre leaders des secteurs avicoles et porcins (Gachet et al 1989).

Tout ceci a évidemment une incidence sur l'organisation du marché. Depuis l'arrêt de toute forme d'organisation collective en élevage porcin, les éleveurs traitent directement avec les bouchers. Ils ont dû s'équiper en moyens de transport ou bien louer les services 
d'un tiers afin d'acheminer les porcs à l'abattoir une fois par semaine. Les prix sont discutés directement entre l'éleveur et le boucher et peuvent varier rapidement en fonction de l'état du marché. Cette voie de commercialisation est la principale pour l'élevage porcin.

Concernant l'élevage avicole, les circuits sont plus variés :

- La vente directe du producteur au consommateur est particulièrement développée, mais difficilement chiffrable.

- La vente sur les marchés est également importante et les quatre principaux aviculteurs de Guyane y sont présents.

- Des accords ont également eu lieu pour la vente dans les supermarchés mais l'instabilité des rapports entre aviculteurs et distributeurs a conduit à la régression de ce mode de commercialisation.

Les oeufs, outre les marchés, sont principalement vendus par l'intermédiaire des "chinois" (épiceries-quincailleries très bien implantées en Guyane).

- Enfin, certains producteurs ont obtenu des contrats avec les collectivités (hôpitaux, écoles, armée), ainsi qu'avec certains restaurants.

Il est important de souligner que la plupart des éleveurs ont souhaité diversifier leur clientèle, avec les objectifs suivants : 1) avoir un contrat avec un supermarché ou une collectivité permettant d'assurer un débouché stable et régulier pour leurs produits. C'est l'objectif de sécurité, 2) se constituer une clientèle de particuliers, soit à la ferme, soit au marché, afin d'écouler le reste de la production, tamponner les fluctuations saisonnières et obtenir une marge plus importante. C'est l'objectif de souplesse et d'adaptabilité.

\section{c / Ajustement de la production à la consommation}

Les importations de viande se font essentiellement sous forme congelée et les oeufs sont réfrigérés. Les consommations en viande de porcs et de volailles ont été respectivement de 2531 tonnes équivalent carcasses (TEC) et de 3413 TEC en 1988. La consommation annuelle de viande de volaille est importante puisqu'elle atteint $38 \mathrm{~kg}$ par habitant en 1988 . Seule la production d'oeufs fait jeu égal avec les importations puisqu'elle atteint 3750 milliers d'oeufs pour 8310 milliers d'oeufs consommés au total (ce chiffre reste très approximatif et on peut sans doute estimer la consommation réelle autour de dix millions d'oeufs). L'évolution du taux de couverture des besoins pour les différents produits a baissé entre 1975 et 1988, surtout à partir de 1986 où les problèmes de structures professionnelles sont apparus. Le taux de couverture pour la viande de porc était de $21 \%$ en 1975 , $23 \%$ en 1981, $55 \%$ en 1984 et a chuté à $13 \%$ en 1988. Pour la viande de volaille, ce taux était de $13 \%$ en 1988 contre $23 \%$ en 1975. Enfin, pour les oeufs, on est passé de $63 \%$ en 1975 à $45 \%$ en 1987 (Bouilly 1989).

\section{d / Perspectives}

Les améliorations à apporter actuellement au sein des filières avicole et porcine concernent tout d'abord la maîtrise technique des ateliers afin de retrouver au moins le niveau d'avant 1986. Des efforts dans ce sens sont d'ores et déjà réalisés avec la reprise de l'activité porcine depuis 1990 , grâce notamment à l'amélioration génétique du cheptel et à la meilleure maitrise des conditions de mise en marché. La création récente d'un service spécifique pour l'élevage (SUAE) à la Chambre d'Ágriculture devrait permettre de pérenniser l'encadrement de la filière, de même que pour l'élevage avicole et cunicole. En outre, la connaissance au jour le jour de l'état et de l'évolution de l'ensemble de ces élevages, voire de l'ensemble du secteur agricole pourrait être tout à fait possible dans un cadre restreint comme celui de la Guyane.

\section{2 / Opération de développement de la filière cunicole}

Amorcée en décembre 1988 avec quelques éleveurs, l'opération d'aide au développement de la filière cunicole a consisté à dynamiser un secteur pour lequel le marché est étroit mais encore ouvert. Par l'importation de reproducteurs et de matériel, il s'agissait de constituer un réseau d'expérimentation et de collecte de données avec plusieurs objectifs :

- étudier les mécanismes et les conditions de développement d'une micro-filière dans le cadre de la diversification des exploitations agricoles ;

- acquérir des résultats et des références sur la production cunicole en Guyane ;

- rendre la production cunicole autonome en Guyane avec la création d'une unité fournissant les animaux de renouvellement.

\section{a / Suivis d'élevages}

Les suivis d'élevages ont concerné 9 éleveurs privés et l'unité de fourniture en reproducteurs de la ferme expérimentale de l'INRA. Le suivi réalisé dans le cadre du réseau a permis l'obtention des résultats suivants : 5,8 lapereaux nés totaux et 5,0 lapereaux sevrés par portée. La mortalité globale (en comptant la mortinatalité) est de $26 \%$ entre la naissance et le sevrage. Les objectifs de 25 à 30 lapereaux sevrés/mère/an, conformément à l'expérience antillaise, semblent donc réalisables moyennant 5 à 6 portées/femelle/an. Il faut tout de même considérer que le contexte commercial est celui d'un marché étroit, segmenté et extrêmement différent de celui de la métropole ou des pays tropicaux où cette spéculation est présente. En effet, la majorité de la clientèle est métropolitaine avec un fort pouvoir d'achat. Ainsi, le prix de vente à la production varie actuellement de 45 à $80 \mathrm{~F} / \mathrm{kg}$ de carcasse. Le lapin frais dans les boucheries de luxe de Cayenne et de Kourou est vendu $89 \mathrm{~F} / \mathrm{kg}$ en 1991. Quant au prix de l'aliment, il varie entre 3,10 et $3,20 \mathrm{~F} / \mathrm{kg}$. La conséquence immédiate du rapport prix aliment du commerce/prix ani- 
mal vendu est qu'il n'existe actuellement pas d'alternative intéressante à l'achat de granulés importés.

\section{b / Essais d'engraissement}

Les résultats obtenus (Ingrand 1990), sachant qu'ils méritent d'être confirmés par des mesures à plus long terme, sont satisfaisants puisque :

- le poids moyen enregistré au sevrage est de $650 \mathrm{~g}$ pour des lapins d'1 mois d'âge ;

- le poids moyen d'1 kg est atteint après deux semaines d'engraissement;

- les $2 \mathrm{~kg}$ de poids vif sont atteints après la 7 e semaine d'engraissement;

- le poids à l'abattage, lequel a eu lieu en moyenne après 9 semaines d'engraissement, est de $2,3 \mathrm{~kg}$. Le gain de poids moyen correspondant est de $26 \mathrm{~g} / \mathrm{j}$.

La consommation individuelle par jour passe d'un peu plus de $50 \mathrm{~g}$ à $140 \mathrm{~g}$, maximum atteint au cours d'oscillations entre 100 et $140 \mathrm{~g}$ après la 4è semaine (figure 3 ). La consommation totale par animal est de $6800 \mathrm{~g}$ en 9 semaines, ce qui correspond à une moyenne de $109 \mathrm{~g} /$ animal $/ \mathrm{j}$. L'indice de consommation sur l'ensemble des animaux est donc de 4,2.

La spéculation cunicole apparaît donc intéressante économiquement, surtout par sa faculté à générer rapidement et régulièrement de la trésorerie. Un facteur important à prendre en compte en Guyane est l'effet de la saison et de la pluviométrie. L'essai décrit ici s'est déroulé principalement durant la grande saison des pluies (avril à juillet). Le taux de mortalité a été très faible durant l'expérimen-

Figure 3. Consommation d'aliment par animal et par jour des lapins de la ferme INRA de Combi (Guyane) durant la période d'engraissement (63 jours).

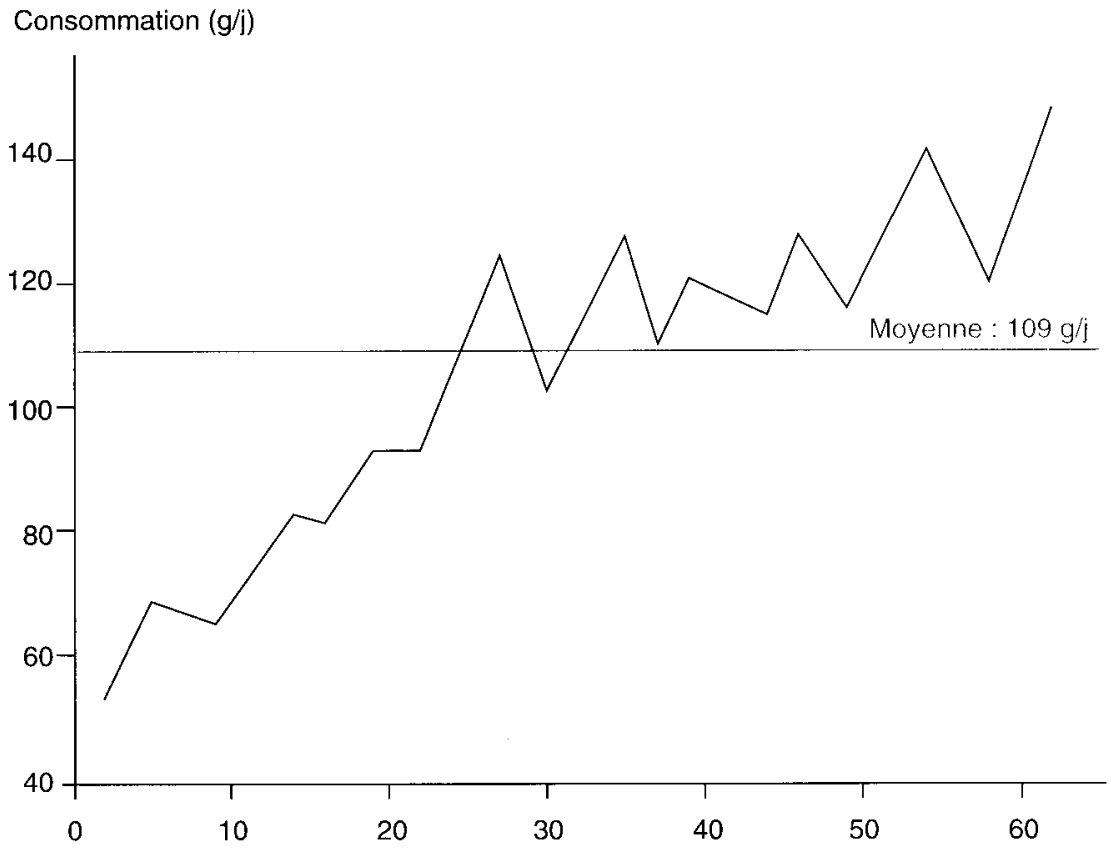

Jours d'engraissement tation malgré les manipulations fréquentes ; ceci se confirme d'ailleurs au niveau des élevages privés où la mortalité apparaît quasiexclusivement durant la phase d'allaitement.

\section{c / Bilan et perspectives}

L'impact de l'opération peut être mesuré à différents niveaux qui ne sont pas tous aisément quantifiables. Par exemple, en deux ans les élevages se sont modernisés et ont augmenté leurs effectifs (tableau 4), de telle sorte que dans plusieurs exploitations, l'atelier cunicole a pris une part significative de l'activité globale (Neyra 1990). Initialement, les neuf éleveurs qui ont reçu des lapins détenaient environ 160 mères, soit 18 femelles en moyenne par élevage. En 1991, six de ces éleveurs (les autres n'ont pas concrétisé leur installation ou ont abandonné la spéculation) totalisent 350 mères, soit une soixantaine en moyenne par élevage. Les éleveurs de ce groupe ont donc intensifié leur activité cunicole mais l'atelier lapins n'est toutefois jamais devenu l'unique spéculation de l'exploitation. En plus de ces élevages se sont créés ou développés d'autres ateliers de tailles diverses (de 5 à 130 mères).

Un autre effet de ces deux années de suivi aura été la meilleure maîtrise de l'atelier suite aux sessions de formation et aux actions spécifiques des agents de Développement. Les éleveurs se sont également progressivement rendu compte de l'intérêt qu'ils ont à se regrouper, notamment pour se placer sur le marché. Le problème principal reste la fixation du niveau des prix qui est encore très variable d'un éleveur à l'autre et d'un client à l'autre. Actuellement, les efforts doivent aller dans le sens d'une organisation des éleveurs qui ne soit plus seulement formelle mais qui puisse être opératoire avec des actions précises et concrètes (groupement des achats d'aliment, planification des ventes, unicité de l'offre vis à vis des clients...).

\section{3 / La valorisation d'espèces sauvages}

Les ressources biologiques du territoire guyanais peuvent constituer une voie intéressante de diversification des productions agricoles (Planquette et al 1989). En effet, la consommation de gibier et de poisson est inscrite dans les habitudes locales. Dans ce contexte, le cabiaï (Hydrochoerus hydrochaeris) est apparu comme un animal tout à fait intéressant. Il a fait l'objet de nombreuses études depuis 15 à 20 ans dans différents pays d'Amérique du Sud et notamment au Venezuela; il pourrait être à l'origine d'une valorisation rapide à l'échelle du département. D'autre part, parmi les poissons, l'atipa (Hoplosternum littorale), siluriforme à plaques osseuses localement apprécié et recherché pour la consommation, est également apparu comme un candidat potentiel dans la perspective d'une valorisation des ressources natu- 
relles régionales. D'après Boujard et al (1988), Luquet et al (1989), Planquette (1989) et Hostache (1991), il pourrait être valorisé dans le cadre d'un développement piscicole local en dépit du fait qu'il intéresse avant tout un marché traditionnel et relativement restreint (estimé à moins d'une centaine de tonnes par an).

\section{1 / Le cabiaï}

\section{a / Caractéristiques biologiques et potentialités d'élevage}

C'est le plus gros rongeur au monde, avec un poids moyen adulte supérieur à $50 \mathrm{~kg}$, une taille atteignant 1 mètre de long et $50 \mathrm{~cm}$ de haut (photo 2). Il est présent en Amérique Centrale et dans la quasi-totalité de l'Amérique du Sud. Le cabiaï est considéré comme l'herbivore non ruminant le plus apte à digérer les fourrages (Gonzalez-Jimenez 1977). En matière de performances de reproduction (tableau 5), les observations réalisées en milieu naturel font état de 1,2 à 1,8 portées/an, à raison de 4 à 6 petits/portée. Une augmentation de la prolificité a pu être obtenue en conditions contrôlées avec des exemples à 8 petits/portée. Le poids à la naissance varie de 1,2 à $2 \mathrm{~kg}$, selon la taille de la portée et le sexe. La maturité sexuelle est atteinte vers 1,5 an, correspondant à un poids de 30 à $40 \mathrm{~kg}$. La durée de la gestation est de 147 jours.

Appelé également cochon d'eau, il est chassé au Venezuela de décembre à Pâques. Traditionnellement, sa viande est consommée durant le carême car elle est assimilée à du poisson par la religion catholique. On la trouve uniquement sous forme salée et séchée et il semblerait que sa valorisation soit bien meilleure en frais (Rexford D. Lord 1990, comm. pers.). La peau est également valorisée dans certains pays (Argentine, Colombie), exploitant le fait qu'elle ne s'étend que dans un sens (propriété particulière au cuir de cet animal).

Le cabiaï peut être affecté par des parasites internes (vers) ou externes (acariens). On peut aisément envisager des traitements préventifs et curatifs dans des conditions d'élevage en semi-liberté à l'aide de produits vermifuges mélangés à un aliment complémentaire de

Tableau 5. Récapitulatif des données concernant le cabiaï en conditions naturelles au Venezuela (d'après Gonzalez-Jimenez 1977).

\begin{tabular}{|lc|}
\hline Durée de gestation (j) & 147 \\
Taille de la portée & 4,73 \\
Nombre de portées par an & 1,83 \\
Poids des mères $(\mathrm{kg})$ & 45 \\
Poids à la naissance $(\mathrm{kg})$ & 1,3 \\
\hline Croissance naissance-abattage $(\mathrm{g} / \mathrm{j})$ & 54 \\
Age à l'abattage (années) & 1,5 \\
Poids à l'abattage (kg) & 30 \\
Rendement carcasse (\%) & 51 \\
\hline Age premières chaleurs (mois) & $12-14$ \\
Age maturité sexuelle mâle (mois) & 16 \\
\hline
\end{tabular}

Tableau 4. Evolution des effectifs de femelles reproductrices dans les élevages de lapins du réseau guyanais. Données du RGA : 1980 : 261 lapines ; $1989: 514$ lapines.

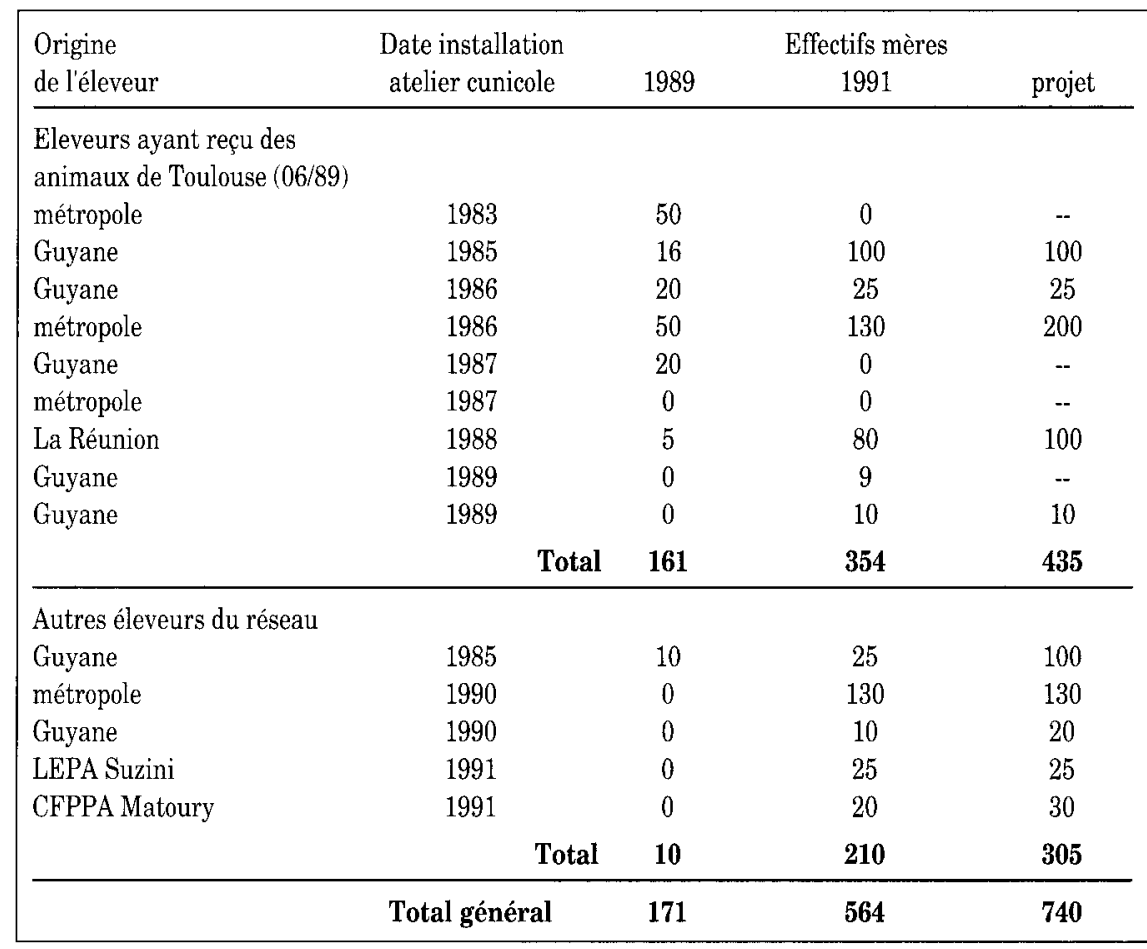

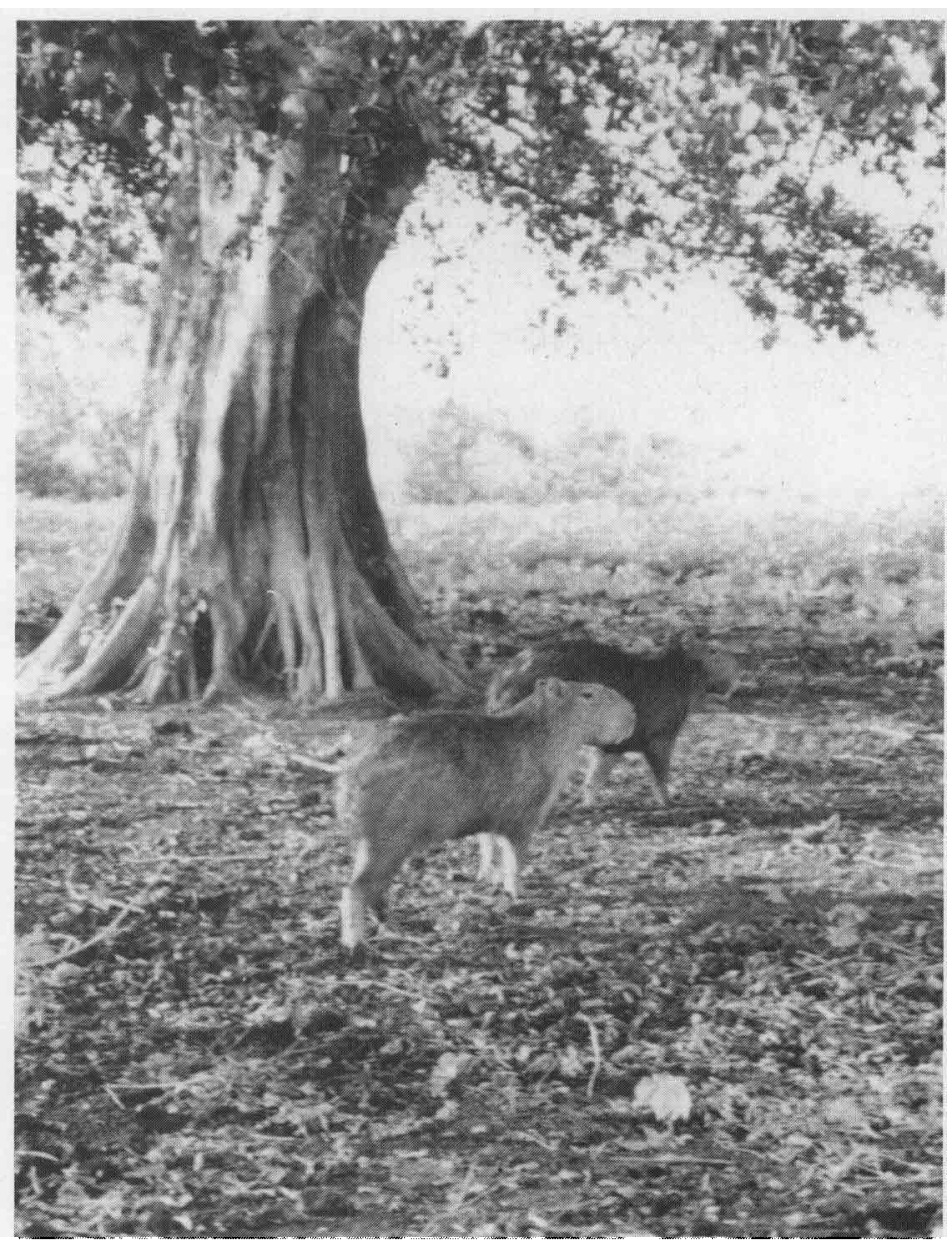

Le cabiaï est un herbivore non ruminant. Adulte, il pèse environ $50 \mathrm{~kg}$ (cliché INRA/S. Ingrand). 
l'herbe pâturée. D'autre part, des études ont montré dès le début du siècle que le cabiaï pouvait être porteur de l'agent pathogène Trypanosoma equinum, et qu'il était fortement déconseillé d'élever ensemble cabiaïs et chevaux (Gonzalez-Jimenez 1977). Son rôle éventuel dans la transmission de la brucellose est également important à prendre en compte, même s'il n'existe aucune preuve actuellement pour affirmer que les cabiaïs peuvent transmettre la brucellose aux bovins ou inversement.

\section{b / Le projet guyanais}

L'importation d'animaux du Venezuela était prévue au début de l'année 1991, la constitution d'un cheptel à partir d'animaux locaux n'ayant pas été possible du fait de la rareté des animaux dans les zones facilement accessibles. Malheureusement, les Services Vétérinaires n'ont pas accordé la dérogation sanitaire indispensable au transfert des cabiaïs du Venezuela vers la Guyane étant donné le risque jugé trop grand d'introduire et de propager la brucellose sur le département, comme cela avait été le cas lors de l'importation de zébus dans le cadre du Plan Vert. Après ce coup d'arrêt porté au projet, les études sur la valorisation de mammifères locaux se sont concentrées sur deux espèces de "cochons sauvages" guyanais : Tayassu tajacu (pécari à collier ou "pakira") et Tayassu albirostris (pécari à lèvre blanche ou "cochon-bois"). Un cheptel a pu être constitué à partir d'animaux capturés en Guyane (généralement des jeunes récupérés par les chasseurs ayant tué la mère). Une collaboration avec le département Elevage des monogastriques de l'INRA, le Museum d'Histoire Naturelle et le CIRAD/EMVT a favorisé la mise en place d'une première structure d'élevage expérimental.

\section{2 / L'atipa (photo 3)}

\section{a / Caractéristiques biologiques et potentialités d'élevage}

L'atipa est un poisson d'eau douce qui a fait l'objet de nombreux travaux de la part des chercheurs de la Station d'hydrobiologie de l'INRA à Kourou. Plusieurs caractéristiques biologiques de l'atipa le font apparaître comme un candidat intéressant pour l'élevage (Hostache et al 1990) : sa croissance rapide (les mâles atteignent un poids de $150 \mathrm{~g}$ en six mois), sa rusticité, sa tolérance à l'hypoxie grâce à une respiration intestinale annexe, son comportement reproducteur particulier qui l'amène à nidifier en surface facilitant ainsi la collecte des oeufs et le contrôle de la production d'alevins.

Les aspects suivants de la production sont actuellement maîtrisés :

- la gestion des pontes en étang à partir d'un petit nombre de géniteurs ou encore de couples isolés en viviers : une femelle de 200 grammes peut pondre jusqu'à 100000 oeufs en 14 pontes, au cours d'une saison de reproduction s'étendant de décembre à mai (Pascal et $a l$, à paraître) ;
- les alevinages pour lesquels la principale difficulté est le contrôle de la prédation par les larves d'odonates (Hostache et al 1990).

Deux points de blocage persistent néanmoins :

- la densité maximale actuellement compatible avec la pleine expression du potentiel de croissance de l'atipa est de 0,5 poisson $/ \mathrm{m}^{2}$. La densité à conseiller en élevage commercial ne semble pas devoir dépasser 4 poissons $/ \mathrm{m}^{2}$.

- la proportion de mâles observée dans les conditions naturelles d'élevage varie de 10 à $80 \%$ (résultats d'alevinages sur trois années). Cela pose pour l'instant le problème de la prédiction de la rentabilité de l'élevage (les mâles étant plus gros que les femelles). L'identification du ou des facteur(s) responsable(s) du sex-ratio devrait permettre d'orienter l'élevage vers la production de monosexes mâles. Préalablement à cette perspective, l'application de températures élevées durant les incubations (30 à $32{ }^{\circ} \mathrm{C}$ ) a déjà permis d'obtenir au cours des deux dernières années des pourcentages de mâles allant de 75 à $95 \%$ (Hostache et al 1992, Hostache et al, à paraître).

\section{b / les perspectives d'élevage en Guyane}

Plusieurs arguments peuvent s'opposer au développement de l'élevage de l'atipa en Guyane :

- le marché guyanais semble limité à moins de 100 tonnes par an : une production d'élevage risquerait d'entraîner la chute des prix sans créer un nombre d'emplois significatif. En outre elle pourrait entraîner une diminution importante de revenu pour les pêcheurs actuels qui utilisent la pêche artisanale de l'atipa comme complément de revenu.

- en ce qui concerne l'exportation, la production guyanaise serait concurrencée par les pêcheries brésiliennes et vénézuéliennes. Dans ces pays, le poisson est vendu à très bas prix $5 \mathrm{~F} / \mathrm{kg}$ contre 45 à $50 \mathrm{~F} / \mathrm{kg}$ en Guyane (Novoa 1982, Tuma 1978).

- le coût de l'aliment importé est prohibitif et il n'existe pas localement de sous-produits agricoles de substitution.

D'autres en revanche sont favorables à ce développement :

- la pêche artisanale guyanaise n'est pas en mesure d'approvisionner totalement le marché local et une grande quantité d'atipas est importée congelée du Brésil ;

- le prix de vente élevé de ce poisson en Guyane : $45 \mathrm{~F} / \mathrm{kg}$ contre 20 à $30 \mathrm{~F} / \mathrm{kg}$ pour les poissons marins ;

- l'essentiel des pêches a lieu entre septembre et janvier, durant la saison sèche et le début de la saison des pluies. Dans un contexte d'élevage, en pratiquant une bonne gestion des pontes, il serait possible de mettre sur le marché du poisson frais durant toute l'année ;

- bien que le marché n'existe pas à l'heure actuelle, ce poisson et l'espèce voisine "l'atipa 
rouge" possèdent toutes les qualités pour constituer un excellent poisson d'aquarium.

La production ne semble donc envisageable que si elle se fait à moindre coût, tant pour les investissements de départ que pour les intrants et le personnel.

Quelques modèles sont susceptibles de réunir ces qualités :

- l'élevage "opportuniste" de l'espèce dans les zones inondées durant la saison des pluies (six mois de l'année) ;

- la production d'oeufs à partir de couples isolés élevés en viviers confectionnés à partir de matériaux locaux (bambous) et permettant de conserver les animaux dont les pontes sont récoltées quotidiennement;

- la production de poissons pour l'aquariophilie : cette spéculation est envisageable dans la mesure où à partir de petits bassins $\left(200 \mathrm{~m}^{2}\right)$ bien conçus et économiques à construire, il est possible de produire chaque mois, de fin novembre à fin juin, plusieurs milliers de poissons d'un poids de 5 à 8 grammes et présentant les qualités pour concurrencer les Corydoras (espèces sud-américaines de la même famille) vendus sur les marchés européens et américains.

\section{4 / Discussion}

En élevage bovin allaitant, dans le cadre du Plan-Vert, l'émergence de stratégies individuelles devant les difficultés d'organisation collective de la production et des marchés a rapidement abouti à un décalage important entre les préoccupations d'une majorité des éleveurs et celles de la Recherche et du Développement. C'est pourquoi le questionnement scientifique élaboré par la station SAD de Guyane à partir de 1986/87 (fin des suivis

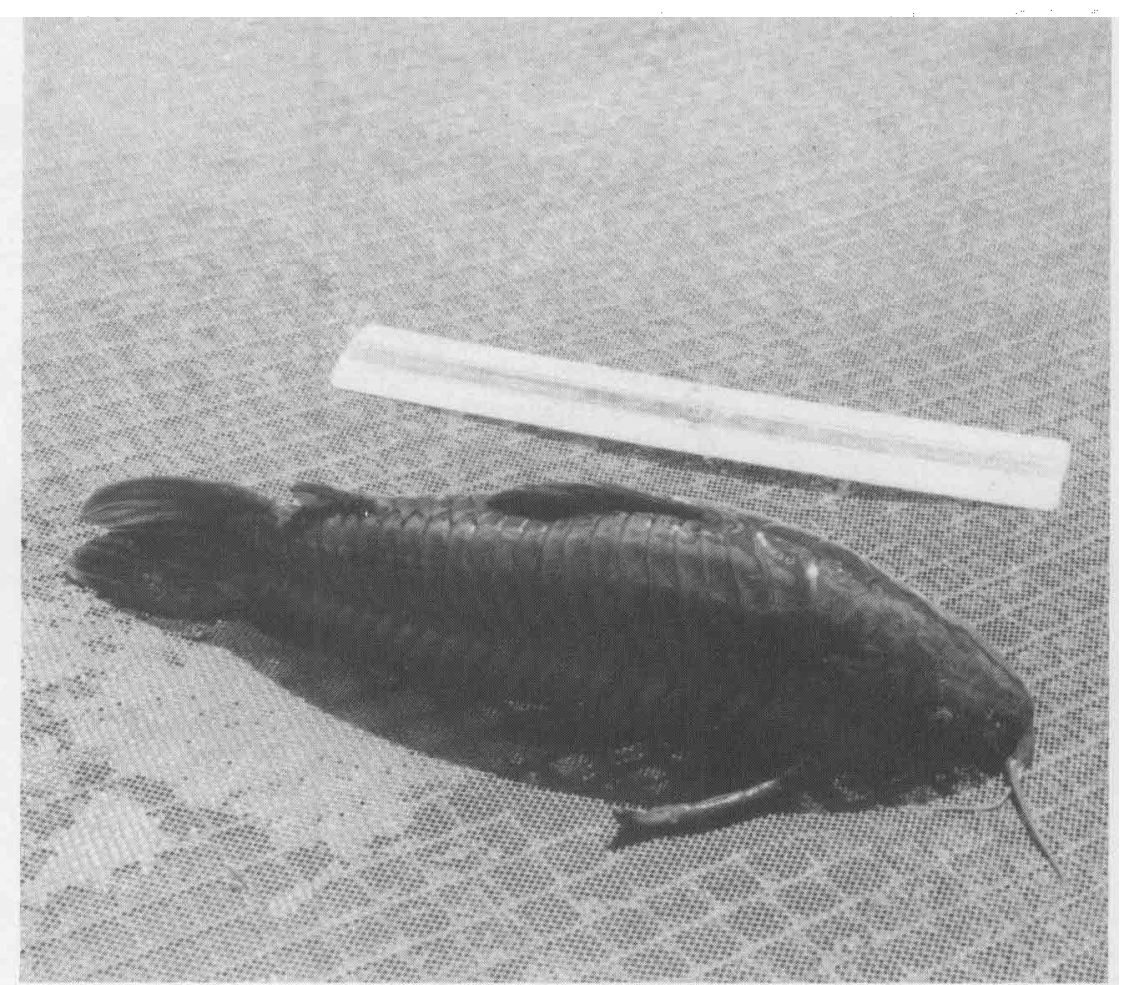

L'atipa est un poisson d'eau douce consommé localement. II est actuellement pêché mais pourrait être produit en pisciculture (cliché INRA / G. Hostache).

d'élevages) a tendu vers l'acquisition de connaissances et d'informations indispensables pour orienter les décisions concernant le Développement agricole. La figure 4 est l'illustration du passage des préoccupations sectorielles de l'équipe (système d'élevage bovinviande exclusivement) à une démarche fondée sur les acquis méthodologiques et les concepts fondamentaux du département SAD (Gachet 1990) : démarche systémique, postulat de complexité des systèmes en cause.

Figure 4. Chronologie des études hors Plan vert réalisées par la Station INRA-SAD en Guyane à partir de 1988 (d'après Gachet 1990).

\begin{tabular}{|c|c|c|c|}
\hline $\begin{array}{cc}1986 & 1987 \\
\text { 1/ Suivis d'élevage } & \vdots\end{array}$ & $\begin{array}{c}1988 \\
\vdots\end{array}$ & \multicolumn{2}{|c|}{1990} \\
\hline 2/ Enquêtes régionales & $\begin{array}{l}\text { 1- Kourou-Macouria } \\
\text { Elaboration de typ }\end{array}$ & $\begin{array}{l}\text { 2- Montsinéry-Macouria } \\
\text { 3- Mana } \\
\text { logies fonctionnelles }\end{array}$ & \\
\hline 3/ Suivis d'exploitations & \multicolumn{2}{|c|}{$\begin{array}{l}\text { Test méthode de } \\
\text { diagnostic (Digrex) } \\
\text { Suivi global : } \\
\text { - acquisitions de références } \\
\text { - méthodologies d'enregistrements, } \\
\text { de restitution }\end{array}$} & $\begin{array}{l}\text { iversification } \\
\text { ons animales }\end{array}$ \\
\hline 4/ Enquêtes thématiques & & Les effets du fumier en sy & $\begin{array}{cr}\text { istème maraîcher } \\
\vdots \\
\text { Relations } \\
\text { consommation / } \\
\text { production } \\
\text { à Kourou }\end{array}$ \\
\hline
\end{tabular}


Les principaux outils utilisés à cet effet ont été les suivis et les enquêtes : enquêtes régionales en 1988 et 1989 , suivis d'exploitations, enquêtes thématiques. Cette démarche systémique a permis d'appréhender la diversité de la réalité agricole guyanaise. L'étape suivante consistera donc à définir des objectifs à atteindre et des méthodes à employer. Il faut alors admettre d'ores et déjà que ces objectifs et ces méthodes puissent être multiples et ajustables. Ainsi, par exemple, le modèle "élevage Plan-Vert" reste un modèle parmi d'autres et ne saurait être généralisable à l'ensemble des producteurs de viande bovine du département. Le cas de la race SantaGertrudis est susceptible de constituer un autre modèle intéressant des catégories d'éleveurs différentes, avec des objectifs et des méthodes pour les atteindre qui sont également différents. On peut reprendre la même logique pour les autres spéculations animales (porcine, avicole et cunicole, aquacole). Les efforts à fournir concernent la définition des actions à entreprendre pour répondre à cette diversité de situations, les enquêtes ayant montré le risque de marginalisation qui existe à vouloir appliquer des systèmes validés dans d'autres contextes.

Ainsi, si nous reprenons l'exemple de l'élevage cunicole, les éléments de la situation initiale qui nous sont apparus importants à prendre en compte dans notre démarche sont les suivants :

- unités existantes de taille réduite au sein d'exploitations diversifiées ;

- aucune organisation collective de la commercialisation;

- prix de vente élevé mais marché limité (20 tonnes de carcasses par an);

- production de qualité convenable mais très fluctuante en quantité ;

- maîtrise technique souvent insuffisante (grande disparité entre les élevages) ;

- renouvellement des reproducteurs non planifié, inorganisé et souvent coûteux ;

- alimentation exclusive aux granulés importés.

L'entrée dans les élevages s'est faite à partir de "l'injection" ponctuelle de produits (animaux, matériel) destinés à lever les contraintes majeures du moment, contraintes exprimées par les éleveurs eux-mêmes et confirmées lors de visites ultérieures. Ce choix a été guidé entre autres par une expérience analogue réalisée aux Antilles.

Après un premier diagnostic de la situation, réalisé en collaboration avec les services du Développement et la coopérative déjà en place, il convenait d'amorcer des actions techniques destinées à formaliser l'encadrement des éleveurs (suivis) et à commencer l'élaboration de références sur la spéculation (suivis, expérimentations). L'objectif était dans un premier temps de sensibiliser les éleveurs aux problèmes et aux spécificités de l'élevage cunicole. Pour ce faire, il était tout à fait opportun d'amorcer parallèlement à l'enregistrement de données, des actions de formation et d'information. Cette voie est essentielle car c'est elle qui joue le rôle de diffusion et de mémorisation. Les sessions de formation ainsi que la création d'un bulletin cunicole s'inscrivent dans cette logique.

C'est la même démarche qui a conduit à préconiser pour l'atipa des projets d'élevages opportunistes de production d'oeufs sous forme de "caviar" local ou de production de poissons pour l'aquariophilie, au lieu d'une production traditionnelle de poissons en bassins.

\section{Conclusion}

Les recherches menées par l'INRA entre 1976 et 1992 liées à l'élevage ont été de deux ordres principaux :

- déterminer les conditions techniques pour atteindre les objectifs fixés dans le cadre du Plan-Vert : comment produire de la viande bovine en Guyane avec du zébu?

- acquérir des connaissances sur les systèmes d'élevage en élargissant le cadre d'étude aux autres espèces animales. Ce thème a rejoint une préoccupation plus générale concernant la nature et la place des spéculations zootechniques au sein de l'exploitation agricole.

Actuellement, en matière d'élevage bovin allaitant, une somme importante de résultats sont disponibles et constituent des références fiables et éprouvées dans le cadre d'un modèle de conduite bien précis, de type intensif, à fort niveau d'intrants. Ainsi, l'échec du Plan Vert n'est sans doute pas d'ordre technique, mais plutôt d'ordre sociologique et financier. A l'image des autres grands projets ayant jalonné le passé de la Guyane, il s'avère illusoire de fonder le développement agricole local sur la seule capacité d'adaptation d'un matériel biologique (animaux, végétaux) à un milieu donné. Ce dernier est en définitive la fraction la plus facilement appréhendable du contexte dans lequel l'activité agricole est pratiquée. Les autres éléments, tout aussi fondamentaux, sont beaucoup plus délicats à définir et à mâ̂triser car ils ne concernent plus (du moins en Guyane), la faculté des acteurs à s'y adapter, mais leur capacité à les générer de toutes pièces et à les gérer sur des pas de temps longs : ces éléments constituent la phase d'organisation et de structuration. Cette étape a été tentée dans la plupart des secteurs d'élevage, mais souvent sans succès, sans doute en grande partie à cause de la rigidité et de la lourdeur des méthodes employées.

Ces dernières années ont vu l'émergence de stratégies individuelles d'adaptation au contexte de crise. A partir de là, des formules souples doivent être mises en place pour reconstituer un semblant de tissu professionnel pour l'ensemble des secteurs des productions animales, pour éviter de voir disparaître ce qui a été péniblement et coûteusement construit.

Enfin, des possibilités existent en matière de valorisation des ressources biologiques 
locales. Pour certaines espèces, des connaissances importantes existent d'ores et déjà, non seulement concernant leur biologie, mais aussi les conditions de leur exploitation dans un contexte d'élevage parfois bien défini (cabiaii), parfois encore prospectif (atipa). Pour d'autres espèces, il reste nécessaire d'effectuer au préalable des recherches plus fondamentales, principalement de physiologie et de comportement social (pécaris).

\section{Références bibliographiques}

Béreau M., Ingrand S., Martin P., Lemaire G., Patient A., 1992. Caractérisation des principales variables d'état de couverts de Digitaria swazilandensis Stent et Brachiaria humidicola (Rendle) Schweickt pâturés en continu par des zébus en Guyane française. Revue Elev. Med. Vet. Pays Trop. , 45, 3-4, 357-366.

Bouilly P., 1989. Etude des filières porcine et avicole en Guyane. INRA-SAD, ENITA de ClermontFerrand. Mémoire de fin d'études. $96 \mathrm{pp}$.

Boujard T., Lebail PY., Planquette P., 1988. Données biologiques sur quelques espèces continentales de Guyane française d"intérêt piscicole. Aquat. Living Resour., 1, 107-113.

Gachet JP., 1990. D'un élevage introduit au système agraire guyanais. In Modélisation systémique et système agraire. Décision et organisation. J. Brossier, B. Vissac, JL. Le Moigne Editeurs. Séminaire SAD St-Maximin, Fév. 1989, 297-319.

Gachet JP., Bourlier F., Thenot MC., 1989. Les conditions de pérennisation de l'activité agricole en Guyane. Stratégie d'approvisionnement d'exploitations spécialisées dans des productions de type industriel et de type traditionnel comparativement à 2 autres exemples d'exploitations de type plus "traditionnel". In : J. Brossier et E. Valceschini éditeurs, Les exploitations agricoles et leur environnement. Essais sur l'espace technique et économique. INRA Paris, 275-288.

Gonzalez-Jimenez E., 1977. Le cochon d'eau : ressource locale pour la production de viande en Amérique tropicale. Rev. Mond. Zoot. 21, 24-30.

Hostache G., 1991. L'atipa: réflexions sur les potentialités d'élevage et la gestion des populations naturelles. Colloque Eco-développement Amazonien, Cayenne. Actes du colloque.

Hostache G., Vallat P., Tessier C., 1990. Maitrise du cycle d'élevage de l'atipa, Hoplosternum littorale. Nature Guyanaise, 4, 33-41.

Hostache G., Pascal M., Kernen M., Teissier C., 1992. Température et incubation chez l'atipa, Hoplosternum littorale (Teleostei, siluriforme). Aqua. Living Resour., 5, 2, 1-9.

Hostache G., Tessier C., Rodolphe F., 1993. Premières observations sur le rôle des conditions thermiques sur le sex-ratio de l'atipa, Hoplosternum littorale (Hancock, 1828). A paraître.

Ingrand S., 1990. L'élevage cunicole en Guyane Française : résultats obtenus durant la période d'engraissement. Affiche. 5èmes journées de la recherche cunicole. Paris. 12-13 décembre 1990.
Ingrand S., Jérome G., 1993. La race SantaGertrudis en Guyane francaise : analyse des performances d'un troupeau expérimental (19741990). Diffusion de la race sur le département. In : M. Vivier, G. Matheron et B. Dedieu éditeurs, La Recherche dans un Plan d'Etat à propos de l'élevage bovin guyanais. Editions CIRAD-EMVT, collection Etudes et Synthèses, Maisons-Alfort.

Ingrand S., Rouville S. de, Matheron G., 1993a. Analyse des essais d'engraissement de taurillons zébus Brahman conduits à l'herbe. Ferme expérimentale de Combi. Guyane française. In : M. Vivier, G. Matheron et B. Dedieu éditeurs, La Recherche dans un Plan d'Etat à propos de l'élevage bovin guyanais. Editions CIRAD-EMVT, collection Etudes et Synthèses, Maisons-Alfort.

Ingrand S., Baynast L.de, Vissac B., 1993b. Analyse des paramètres de la reproduction du troupeau allaitant de la ferme de St-Elie. Guyane francaise. In : M. Vivier, G. Matheron et B. Dedieu éditeurs, La Recherche dans un Plan d'Etat à propos de l'élevage bovin guyanais. Editions CIRAD-EMVT, collection Etudes et Synthèses, Maisons-Alfort.

Ingrand S., Vissac B., Grude A., 1993c. L'Association de Mise en Valeur Agricole (AMVA) de St-Elie. Bilan de fonctionnement et résultats. In : $M$. Vivier, G. Matheron et B. Dedieu éditeurs, La Recherche dans un Plan d'Etat à propos de l'élevage bovin guyanais. Editions CIRAD-EMVT, collection Etudes et Synthèses, Maisons-Alfort.

INRA, 1981. Prairies guyanaises et élevage bovin. Actes du colloque inter-instituts INRA, ORSTOM, GERDAT. Cayenne-Suzini, 15-16 décembre 1981. Editions INRA Paris, collection Les colloques de I'INRA, 24.

INRA, 1985. Systèmes d'élevage herbager en milieu équatorial. Actes du séminaire, Cayenne. 9-10 décembre 1985. Editions INRA, Paris.

Lebouteiller V., Leroux P., Rannou T., 1987. Suivi d'élevages zébus guyanais en 1986. Bilan technique. INRA SAD Kourou, SUAD/Chambre d'agriculture de la Guyane, CEBG. 27 p.

Luquet P., Boujard T., Planquette P., Moreau Y., Hostache G., 1989. The culture of Hoplosternum littorale : state of the art and perspectives. Advances in Tropical Aquaculture. Tahiti, Feb. 20 - March 4. 1989. AQUACOP IFREMER. Actes du colloque, 511516.

Neyra N., 1990. Le système d'élevage cunicole en Guyane française. L'impact d'une opération d'appui au développement. INRA SAD, CNEARC. Mémoire de fin d'études, $125 \mathrm{pp}$. 
Novoa D., 1982. Los recursos pesqueros del rio Orinoco y su exploitacion. Corporation venezolana de Guyana. Div. de desarollo agricola, 151-157.

Pascal M., Hostache G., Tessier C., Vallat P. Cycle de reproduction et fécondité de l'atipa, Hoplosternum littorale (Hancock, 1828) en milieu semi-naturel. Aqua. Living Resour., sous presse.

Planquette P., 1989. Données sur la biologie générale et la reproduction des atipas (Hoplosternum spp). Nature guyanaise, 2, 8-13.

Planquette P., Béreau M., Gachet JP., 1989. Note de présentation pour l'ouverture d'un nouvel axe de recherches au groupe de Guyane : définition des bases biologiques nécessaires à la mise en oeuvre d'élevages contrôlés de certains mammifères et oiseaux de la faune locale. INRA. Groupe Guyane. $18 \mathrm{pp}$.

Tuma Y.S., 1978. Contribuicao para o conhecimento da biologia do tamuata, Hoplosternum littorale (Hancock, 1828), Eigenmann \& Eigenmann, (Pisces callichthydae) da Ilha de Marajo, Para, Brasil. B.FCAP, Belem. Bull. da faculdad de Cienc. Agrar. Para, 10, 59-76.

Vissac B., 1992. Les fusées et la forêt amazonienne cacheraient-elles des indiens ? En guise de bilan d'un Plan d'Etat. In : Courrier de la cellule environnement, 16. INRA Paris.

\section{Summary}

Animal production in French Guiana. Results obtained with domestic species and investigations conducted on two wild local species

In French Guiana, zootechnical research run by INRA, within the developpement program called "Plan-Vert" initiated in 1975 , were specially focused on cattle rearing (zebu Brahman), in order to increase local beef meat production.

Most of the technical datas concern reproductive performances under tropical climate and rearing of bulls on pasture between weaning ( 9 to 10 months of age) and slaughtering (24 to 30 months of age). In addition, the control of performances of private herds from 1985 to 1987 , have contributed to analyse "on-farms" technical results.This has been the first step towards a typology based on management practices of the farmers.

Investigations and studies about other species (pigs, poultry, rabbits) have led to conclude, regarding the number of farmers involved, that beef production is not prevailing in French Guiana. The main characteristic of breeding systems is the diversification of the productions (one farm : many species), in order to adapt to the local market, and to satisfy their necessity of cash-flow.

In this situation, where diversification of rearing activities is valuable, local wild species could turn out to be an interesting way to valorize the natural biological resources of French Guiana. Up to now, two species have been studied in this respect, a local rodent : the cabiaï (Hydrochoerus hydrochaeris), and a freshwater fish : the atipa (Hoplosternum littorale).

In conclusion, considerations about necessary conditions to develop sustainable breeding systems in French Guiana are presented. They stress the idea that taking into account economical and sociological aspects is of a special importance in the guianese context.

INGRAND S., HOSTACHE G., 1993. L'élevage en Guyane. Bilan des travaux réalisés sur les espèces domestiques et sur deux espèces sauvages autochtones. INRA Prod. Anim., 6 (5), 319-332. 